\title{
Mechanistic Insights into the Meerwein-Ponndorf-Verley \\ Reaction and Relative Side Reactions over MgO in the Process of \\ Ethanol to 1,3-Butadiene: A DFT Study
}

Minhua Zhang ${ }^{\mathrm{a}, \mathrm{b}}$, Ruishen $\mathrm{Li}^{\mathrm{a}, \mathrm{b}}$, Yufei $\mathrm{Wu}^{\mathrm{a}, \mathrm{b}}$, Yingzhe $\mathrm{Yu}^{\mathrm{a}, \mathrm{b}, *}$

${ }^{a}$ Key Laboratory for Green Chemical Technology of Ministry of Education,

R \& D Center for Petrochemical Technology, Tianjin University, P R China

${ }^{\mathrm{b}}$ Collaborative Innovation Center of Chemical Science and Engineering (Tianjin)

Tianjin 300072, P R China

* Corresponding author. Tel.: +86-22-27405972; fax: +86-22-27406119.

E-mail address: yzhyu@tju.edu.cn (Yingzhe Yu)

\section{Index}

Table S1 Results of conduction band centers of $\mathrm{Mg}$ on $\mathrm{MgO}(100)$ and $\mathrm{MgO}(110)$ surfaces.

Table S2 Adsorption parameters of $\mathrm{CO}_{2}$ on $\mathrm{MgO}(100)$ and $\mathrm{MgO}(110)$ surfaces.

Table S3 Mulliken population (|e|) of ethanol and acetaldehyde on $\operatorname{MgO}(100)$ and $\mathrm{MgO}(110)$ surfaces.

Figure S1 PDOS diagram on $\mathrm{MgO}(100)$ and $\mathrm{MgO}(110)$ surfaces.

Figure $\mathbf{S 2} \mathrm{CO}_{2}$ configurations on (a) $\mathrm{MgO}(100)$, (b) $\mathrm{MgO}(110)$.

Figure S3 The adsorption configurations of reactants and intermediates, (a-e), on $\mathrm{MgO}(100)$ and (f-i) on $\mathrm{MgO}(110)$ surface; green, $\mathrm{Mg}$ atoms; red, $\mathrm{O}$ atoms; black, $\mathrm{C}$ atoms; white, $\mathrm{H}$ atoms. 
Table S1 Results of conduction band centers of $\mathrm{Mg}$ on $\mathrm{MgO}(100)$ and $\mathrm{MgO}(110)$ surfaces.

\begin{tabular}{|c|c|c|}
\hline Surface & $\operatorname{MgO}(100)$ & $\operatorname{MgO}(110)$ \\
\hline $\int_{\epsilon_{F}}^{\infty} \rho(\epsilon) \epsilon d \epsilon(\mathrm{Ha})$ & 5.30 & 3.18 \\
\hline $\int_{\epsilon_{F}}^{\infty} \rho(\epsilon) d \epsilon$ & 35.09 & 30.10 \\
\hline$E^{*}(\mathrm{Ha})$ & 0.15 & 0.10 \\
\hline
\end{tabular}

Table S2 Adsorption parameters of $\mathrm{CO}_{2}$ on $\mathrm{MgO}(100)$ and $\mathrm{MgO}(110)$ surfaces.

\begin{tabular}{|c|c|c|}
\hline Parameters & $\operatorname{Mg}(100)$ & $\operatorname{Mg}(110)$ \\
\hline$E_{a d s}(\mathrm{eV})$ & -0.62 & -2.87 \\
\hline$d\left(\mathrm{C}-\mathrm{O}_{\mathrm{s}}\right)(\AA)$ & 1.45 & 1.34 \\
\hline$d(\mathrm{C}-\mathrm{O})(\AA)$ & 1.26 & 2.08 \\
\hline$d\left(\mathrm{Mg}_{\mathrm{s}}-\mathrm{O}\right)(\AA)$ & 2.20 & 128.60 \\
\hline$\angle\left(\mathrm{O}_{1}-\mathrm{C}-\mathrm{O}_{2}\right)\left(^{\circ}\right)$ & 133.07 & 1.29 \\
\hline
\end{tabular}


Table S3 Mulliken population (|e|) of ethanol and acetaldehyde on $\mathrm{MgO}(100)$ and

$\operatorname{MgO(110)~surfaces.~}$

\begin{tabular}{|c|c|c|c|}
\hline \multirow{2}{*}{ Atom } & Ethanol on & Acetaldehyde on & Acetaldehyde on \\
& $\mathrm{MgO}(100)$ & $\mathrm{MgO}(100)$ & $\mathrm{MgO}(110)$ \\
\hline $\mathrm{O}$ & -0.614 & -0.379 & -0.588 \\
\hline $\mathrm{C}_{\mathrm{e} 1}$ & 0.102 & 0.256 & 0.325 \\
\hline $\mathrm{C}_{\mathrm{e} 2}$ & -0.333 & -0.381 & -0.383 \\
\hline $\mathrm{H}$ & 0.129 & 0.173 & 0.200 \\
\hline
\end{tabular}




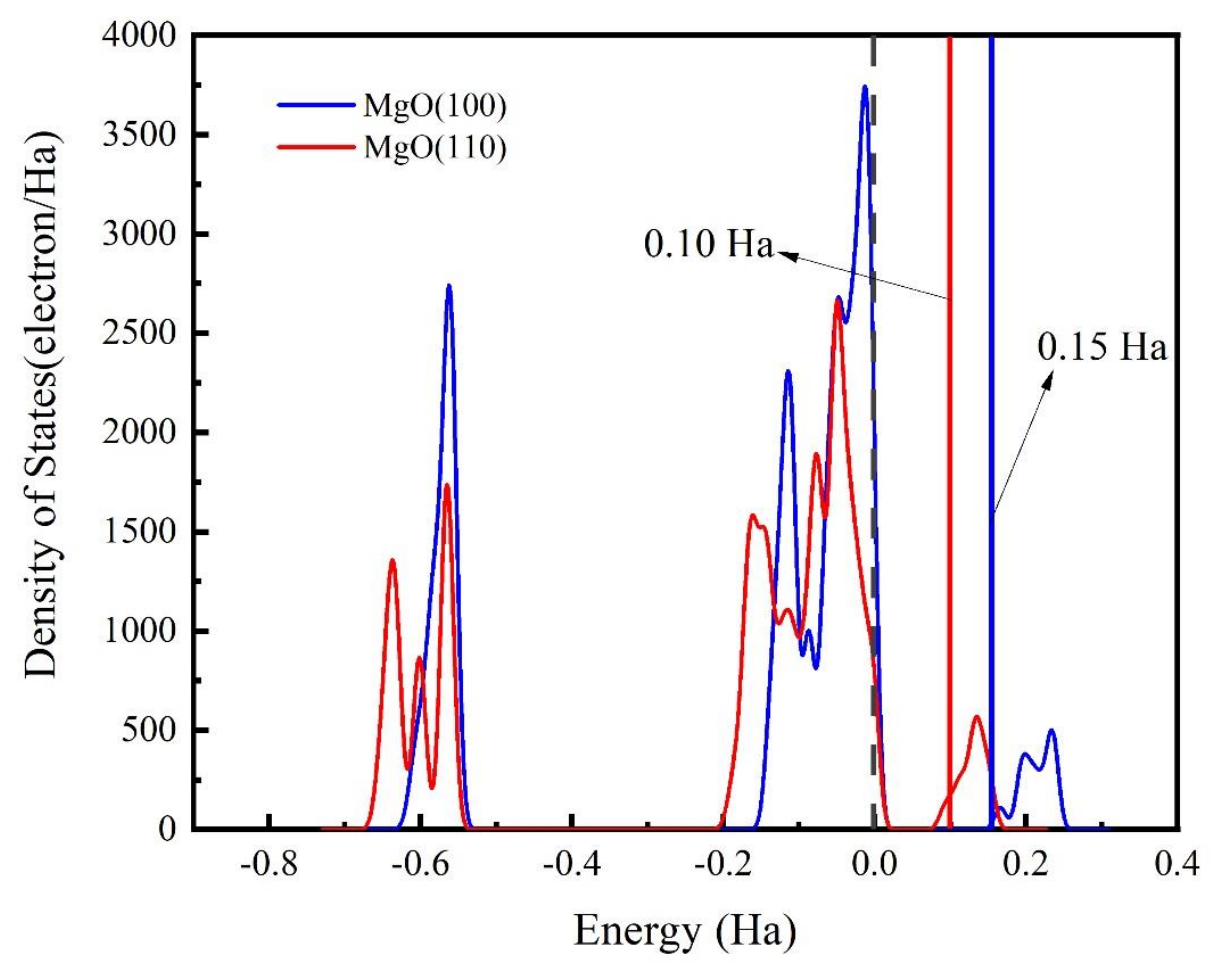

Figure S1 PDOS diagram on $\mathrm{MgO}(100)$ and $\mathrm{MgO}(110)$ surfaces.
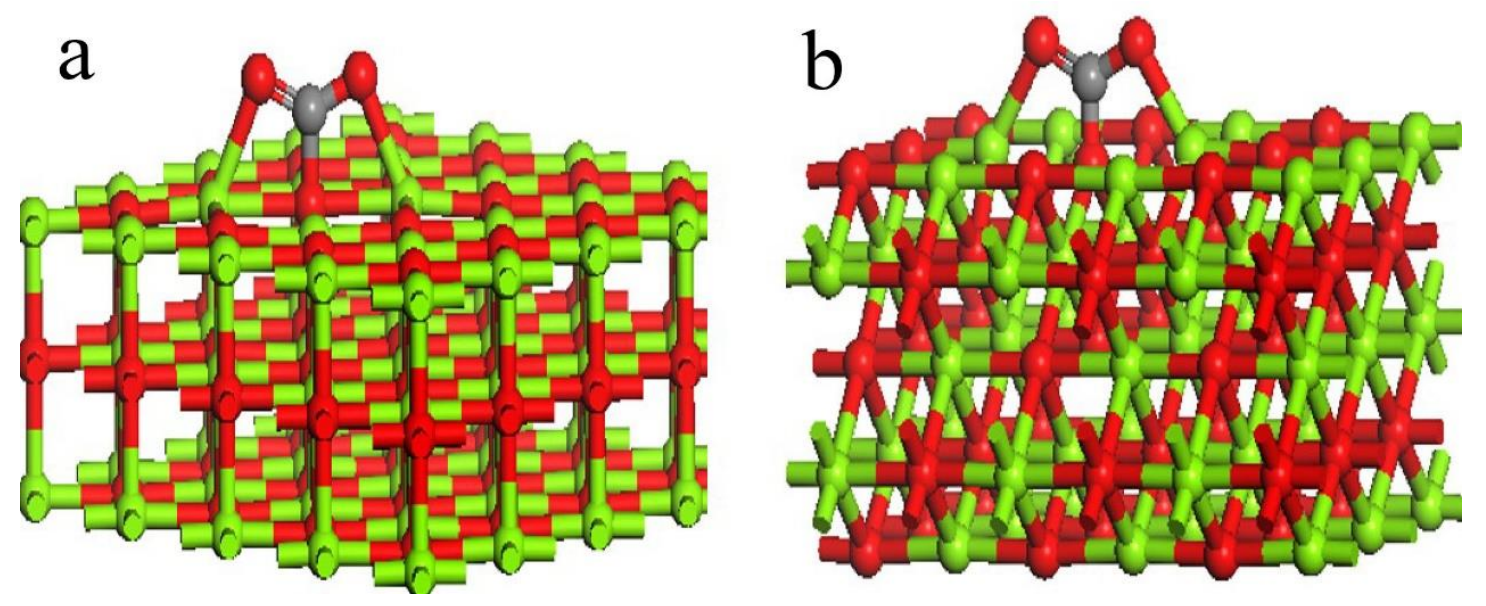

Figure $\mathrm{S} 2 \mathrm{CO}_{2}$ configurations on (a)MgO(100), (b)MgO(110). 


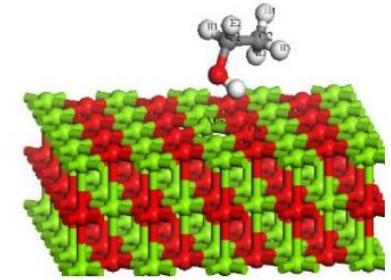

a

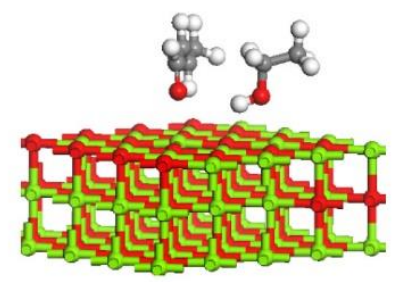

d

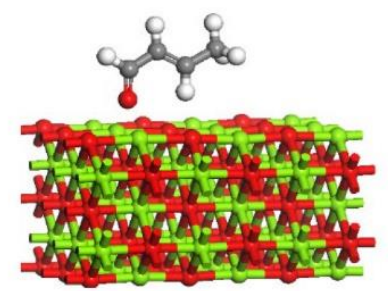

g

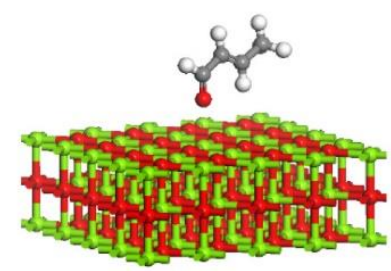

b

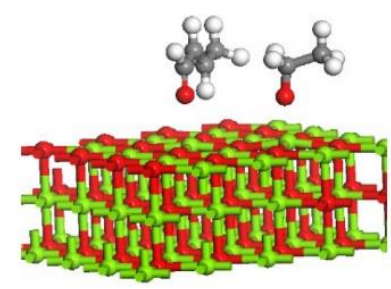

e

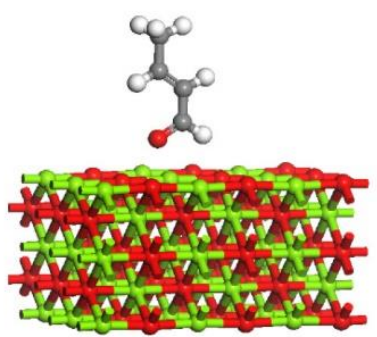

h

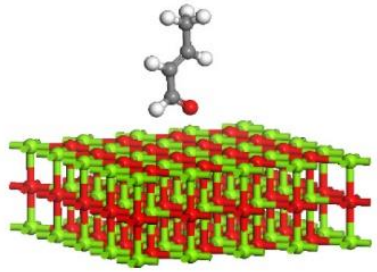

c

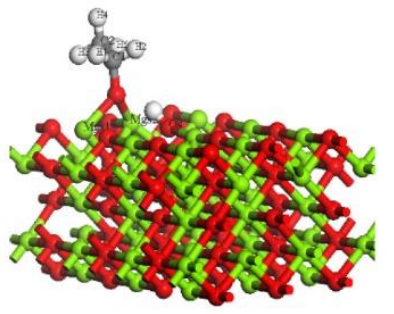

f

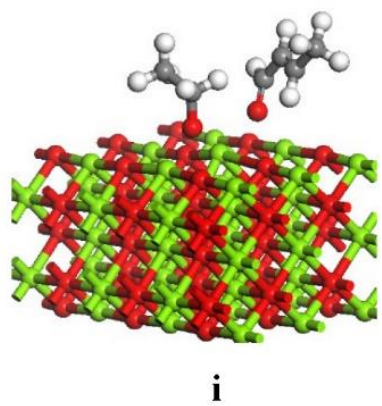

Figure S3 The adsorption configurations of reactants and intermediates, (a-e), on MgO(100) and (f-i) on MgO(110) surface; green, Mg atoms; red, O atoms; black, $\mathrm{C}$ atoms; white, $\mathrm{H}$ atoms. 